\title{
Building Simulation has set up a new goal to publish papers quickly
}

\author{
Xudong Yang ( $\square)$ \\ Editor-in-Chief \\ Department of Building Science, School of Architecture, Tsinghua University, Beijing 100084, China
}

๑) Tsinghua University Press and Springer-Verlag Berlin Heidelberg 2014

First, I am honored to announce that the following distinguished researchers have agreed to join the editorial board of Building Simulation journal:

William P. Bahnfleth, The Pennsylvania State University, USA Bert Blocken, Eindhoven University of Technology, The Netherlands Philip Jones, Cardiff University, UK

Ron Judkoff, National Renewable Energy Laboratory, USA

John Z. Lin, City University of Hong Kong, China

Bing Liu, Pacific Northwest National Laboratory, USA

Bjarne W. Olesen, Technical University of Denmark, Denmark

We thank them for their willingness to serve the Journal, and look forward to a pleasant collaboration many years ahead.

Since 2013 (Vol. 7), the Journal has changed the publishing frequency from quarterly to bi-monthly, and significantly increased the page budget. Some authors worried whether the publishing speed could be affected. After one year's operation, I am proud to report that our publishing speed becomes even quicker!

From day one, publishing quickly has been on top of our priority list. Our original target was that for a tworound double-blind review process, the time from a paper's first submission to its final disposition should be less than six months. With a joint effort of our reviewers, authors, editorial office, and publishers, we now intend to further shorten that to four (4) months. Most of that time is given to reviewers to carefully review, and authors to carefully revise the manuscripts. With the help of Springer's Online First program, most authors will be surprised to see how fast their research results can turn into archival publications in our journal.

In order to attract more high quality submissions, the Journal will also slightly expand its scope. Previously, we require that all submissions be explicitly related to simulations under all five columns. While this requirement will still be valid, we will also consider papers that are based on experimental work. We believe that high quality experimental data are key part of building simulation domain. Moreover, we always welcome review papers or position articles dealing with various aspects of building simulation science and technologies.

Meanwhile, let me re-iterate that the quality control will not in any way be affected by the above changes. In fact, we have further tightened the quality control by applying higher standards in accepting papers. Review comments are as much helpful to authors to improve the paper quality. And yet, we stick to our principle to welcome submissions from all over the world. All papers are treated equally and fairly by our double-blind paper review system, and we believe that expert reviewers' comments are the utmost opinion in our decision making process.

Please come and visit Building Simulation often. 